Fecha de recepción: julio 2021

Fecha de aprobación: agosto 2021

Fecha publicación: septiembre 2021

\section{Prólogo. Arte, comunicación y política: experiencias y expresiones desde Latinoamérica}

Natalia Aguerre ${ }^{(1)}$ y Janneth Arley

Palacios Chavarro ${ }^{(2)}$

Resumen: Se reflexiona en clave crítica y desde distintas perspectivas sobre el conjunto de experiencias, expresiones y prácticas que se asientan en el reconocimiento de la función social del arte, el diseño y la comunicación en el compromiso con la ciudadanía, la libertad de expresión, la defensa de los derechos, la construcción social de realidad, la intervención en el espacio público, el cambio de perspectiva del espectador y los modos de narrar y contar nuestro actual contexto marcado por la Pandemia del Covid-19.

Palabras clave: arte - comunicación - política.

[Resúmenes en inglés y portugués en las páginas 14-15]

(1) Natalia Aguerre. Licenciada en Comunicación Social y Periodismo. Doctora en Comunicación. Profesora de grado y posgrado en la Facultad de Periodismo y Comunicación Social de la Universidad Nacional de La Plata; la Facultad de Diseño y Comunicación de la Universidad de Palermo y la Universidad Arturo Jauretche. Integrante de equipos de investigación -UNLP, CLACSO-, que abordan temas sobre arte y política. Fundraiser y Gestora en Comunicación del Centro de Arte Experimental Vigo. Correo electrónico: aguerre.natalia@yahoo.com

(2) Janneth Arley Palacios Chavarro. Doctoranda en Comunicación Audiovisual, Publicidad y Relaciones Públicas de la Universidad Complutense de Madrid. Magíster en Administración de la Universidad Nacional de Colombia. Comunicadora Social de la Universidad Central. Profesora de tiempo completo, investigadora de la Facultad de Ciencias de la Comunicación de la Fundación Universitaria Los Libertadores, Bogotá (Colombia). Líneas de investigación: comunicación, cultura y tecnología; Comunicación; Comunicación Estratégica; Comunicación Organizacional, Publicidad y Relaciones Públicas. Correo electrónico: japalaciosc@libertadores.edu.co 
El presente volumen fue destinado a reflexionar en clave crítica y desde distintas perspectivas sobre el conjunto de experiencias, expresiones y prácticas que se asientan en el reconocimiento de la función social del arte, el diseño y la comunicación en el compromiso con la ciudadanía, la libertad de expresión, la defensa de los derechos, la construcción social de realidad, la intervención en el espacio público, el cambio de perspectiva del espectador y los modos de narrar y contar nuestro actual contexto marcado por la Pandemia del Covid-19.

Estos elementos han contribuido a configurar un nuevo régimen de las artes -según el concepto de Jacques Rancière (2010)-, abriendo a una nueva etapa en los modos de producir, exhibir y conceptualizar las manifestaciones estéticas otorgando, además, espacios y articulaciones con formas de activismo político, producción económica e investigación científica.

Se buscó ampliar la perspectiva dialógica entre los tres aspectos propuestos para este número, al proponer el arte como un escenario dinámico de comunicación de la realidad cultural y de los fenómenos sociales, que permite entrever apuestas políticas y modos de ver y resignificar la realidad y el "orden social".

Pensar el arte, la comunicación y la política desde sus relaciones y tensiones y a partir de las posibilidades para construir, reconocer e interpretar diferentes sentidos y realidades, nos abre un escenario amplio para traer a reflexión nuestra cultura latinoamericana, heterogénea, híbrida y dinámica y propiciar un diálogo que exponga otras formas de observar, pensar, sentir, relacionar y transformar el mundo. El arte, nos da la posibilidad de crear "otra forma de comunicar", al decir de Gruner (2000) "el arte puede (...) generar alternativas de otra comunicación: una comunicación que, por así decir, atente contra la lógica férrea de la falsa "unidad" de lo visible, que gobierna actualmente, permitiendo que aflore la pregunta, la interrogación crítica sobre los enigmas del mundo (...). Una comunicación que restituya lo visible como conflicto de la visión, la cultura como campo de batalla entre lo "comunicable" y lo "incomunicable".

En este marco, la propuesta que se llevó a cabo en colaboración con la Fundación Universidad Los Libertadores, Colombia pretendió abrir el debate en torno a los desafíos que presentan estas acciones en la actualidad para América Latina, considerando la dimensión política del arte y la comunicación desde sus entramados, relaciones y contextos. Desde esta perspectiva se hace notable en el presente número el interés de varios autores por analizar y develar el papel de las tecnologías de información, comunicación y ahora, de relacionamiento, particularmente, en el contexto de las transformaciones sociales y culturales que se viven en diferentes lugares de nuestra región.

Las reflexiones aquí desarrolladas -que no pretenden ser conclusivas-, permitirán aportar algunas líneas para pensar dicha vinculación, a partir de subrayar la importancia de edificar un análisis social, crítico, complejo y políticamente situado de las prácticas artísticas desde una perspectiva transdisciplinar.

Dicho Cuaderno está compuesto por escritos de:

Silvia Lorena Álvarez León y Kléver Rolando Samaniego Pesantez: El artículo indaga sobre el confinamiento, el distanciamiento social y los juegos en dispositivos electrónicos que están constituyéndose en un obstáculo para la reactivación de las actividades físicas en tiempos post-pandemia. Por esa razón proponen iniciar con un pensamiento prospec- 
tivo para disminuir los efectos negativos que podrían afectar al momento de reactivar las actividades físicas realizando una exploración comunicacional por los procesos intersubjetivos existentes en las Jornadas Deportivas Vacacionales de Sígsig-Ecuador, considerada la más constante y antigua del Ecuador.

Blas Arrese Igor: Aborda la dramaturgia de la recepción como un área de vacancia para las investigaciones que cruzan comunicación social y teatro. El artículo se adentra en "Jamlet de Villa Elvira”, experiencia artística a partir del clásico shakesperiano, llevada adelante por pibes y pibas de un grupo de teatro de un barrio periférico de la ciudad de La Plata (Argentina). Nació como parte del trabajo de campo para la tesis doctoral del artista e investigador Blas Arrese Igor, para luego rebasar los límites académicos promoviendo la generación del Grupo de Teatro Oriyero. La experiencia llevada a cabo en Villa Elvira nos permite pensar las potencias del arte en los márgenes, en las periferias

Mónica Baquero Gaitán y Nohra Ramírez Herrera: Trabajan sobre los cambios que afronta la publicidad debido al impacto de la revolución tecnológica, la exigencia de efectividad y la urgencia de formación de profesionales con competencias aún por definir. El artículo se configura como un proyecto cuya intención es la comprensión de los retos que trae consigo la cuarta revolución industrial en el ejercicio de la profesión y en el estudio del fenómeno publicitario contemporáneo. Para ello, retoma las voces de publicistas de Iberoamérica y se indaga si la industria publicitaria como industria creativa está por reinventarse o si se enfrentan, el efecto Kodak o Blockbuster, propios de la obsolescencia del negocio.

Laura Camila Bautista Bautista: En este artículo se reflexiona sobre los hallazgos de la investigación realizada, a partir del análisis de la forma de comunicación gráfica del grupo de Rock fusión bogotano "Calle e la belleza", desde su nacimiento en 2014 hasta el 2020. El fin principal de la investigación es lograr determinar si el proceso comunicacional mediado por el vestuario, el arte visual, las letras y los recursos gráficos que plantea el grupo, van acorde con lo que proyecta, desde la apropiación y creación de la música fusión hasta el desarrollo cultural para hacer de la estructura simbólica y su propio discurso un medio de expresión.

Andrea Forero Hurtado y Andrés Torres Poveda: A partir del estudio de la intervención de la alcaldía de Bogotá en 2016 a una zona del centro de la ciudad, deprimida por fenómenos de narcotráfico, prostitución, miseria e ilegalidad, conocida como El Bronx o La Ele, se desarrollaron acciones para la atención de las personas que ocupaban el sector; algunas centradas en el arte, como la obra de teatro musical "Gloria, un canto a la vida”. De allí la investigación "Del territorio de la calle al territorio de la escena teatral”. La riqueza de los relatos sobre las prácticas y los saberes de La Calle como territorio, es decir como espacio de la construcción de sentidos, dieron pie a este artículo. El tema principal en este escrito, antes que el hecho teatral, es el fenómeno de habitabilidad de calle descubierto a través de las narrativas y que suscitaron reflexiones y tímidas propuestas de manejo.

Diana Tamara Molina Reyes: Analiza las prácticas de gestión comunicacional de Mashasha, una casa de diseño independiente de indumentaria de la ciudad de Quito, para observar sus estrategias comunicativas de posicionamiento en el mercado. Mediante una investigación de tipo mixto, que contempla entrevistas a su creadora y encuestas al público objetivo, se determina que existen importantes brechas en la gestión de marca por falta de conocimiento de su creadora y los costos de este proceso, lo cual genera un escaso re- 
conocimiento de esta casa de diseño aun cuando el público tiene conocimiento sobre este ámbito comercial y que lleva, finalmente, a su desaparición.

Victoria Nannini: Su estudio aborda las prácticas relacionadas al consumo de indumentaria de moda en jóvenes a través de medios digitales y plataformas virtuales que se ven influenciadas por la cultura pop japonesa. De esta forma, es posible detectar prácticas de consumo que samplean, mezclan y replican vestigios culturales japoneses que van desde el animé, pasando por lo kawaii hasta el uso de kanjis japoneses, junto a otras estéticas y estilos de moda y, por consiguiente, comunican nuevas construcciones de subjetividades respecto de la apariencia, la selección de marcas, el género, entre otros aspectos.

Lilia Nogueira Calcagno Horta: El artículo expone y examina la estética afectiva de Isao Takahata, abordando sus peculiaridades e investigando cómo recopila sus obras. Para eso, utilizaremos autores de la comunicación que estudian la estética de las producciones culturales, como Morin, Parret, Serres y Lotman, así como los sesgos de la industria del anime cultural, que serán abordados a través de los pensamientos de Tsugata, Luyten, Cavalaro y Takahata.

Dariane Lima Arantes y Pedro de Assis Pereira Scudeller: La investigación propone, a partir de un análisis comparativo entre el bestseller Little Fires Everywhere y su adaptación para televisión, tensar los sentidos relacionados a marcadores de género, raza y de clase presentes en sus narrativas. Buscamos comprender cómo se incorporan y negocian las narrativas de la diferencia en estas obras, a partir de reflexiones sobre la cultura remix, e identificar en qué medida las narrativas revelan regímenes de visibilidad y se convierten en operadoras de disenso, estableciendo una política de diferencia respecto a las representaciones femeninas. Pensamos en las intersecciones entre experiencia estética, cultura mediática y representaciones de la diferencia.

Cyntia Valladares: En el marco de la lucha por la legalización del aborto en Argentina -específicamente en la ciudad de Buenos Aires entre el 2018 y el 2020-, el símbolo de la Campaña Nacional por el Derecho al Aborto Legal, Seguro y Gratuito representado por el pañuelo verde excedió su lugar de origen expandiendo y generando nuevas ocasiones, modos de uso y ampliando sus significados. El presente artículo indaga sobre el lugar que ocupó el pañuelo verde en la lucha por la legalización del aborto, haciendo foco en las características de esta prenda política que incidieron en la problematización del género, desde su actuar performático.

\begin{abstract}
It reflects critically and from different perspectives on the set of experiences, expressions and practices that are based on the recognition of the social function of art, design and communication in the commitment to citizenship, freedom of expression, defense rights, the social construction of reality, intervention in public space, the viewer's change of perspective and the ways of narrating and telling our current context marked by the Covid-19 Pandemic.
\end{abstract}

Keywords: art - communication - politics. 
Resumo: Reflete-se de forma crítica e sob diferentes perspectivas sobre o conjunto de experiências, expressões e práticas que se baseiam no reconhecimento da função social da arte, do design e da comunicação no compromisso com a cidadania, a liberdade de expressão, a defesa dos direitos, a construção social da realidade, a intervenção no espaço público, a mudança de perspectiva do espectador e as formas de narrar e contar o nosso contexto atual marcado pela Pandemia Covid-19.

Palavras chave: arte - comunicação - política.

[Las traducciones de los abstracts fueron supervisadas por el autor de cada artículo] 